\title{
Adolescentes que vivem com necessidades especiais de saúde: Análise do discurso emancipatório
}

\begin{abstract}
Andressa da Silveira ${ }^{1}$ e Eliane Tatsch Neves ${ }^{2}$
${ }^{1}$ Departamento de Ciências da Saúde, Universidade Federal de Santa Maria, Brasil.

Docente da Universidade Federal de Santa Maria, Palmeira das Missões, Brasil | andressadasilveira@gmail.com | https://orcid.org/0000-0002-4182-4714

${ }^{2}$ Departamento de Enfermagem, Universidade Federal de Santa Maria, Brasil. Docente da

Universidade Federal de Santa Maria, Santa Maria, Brasil | eliane.neves@ufsm.br | https://orcid.org/0000-0002-1559-9533

Resumo: Introdução: Adolescentes com necessidades especiais de saúde (CRIANES) necessitam de cuidados de saúde para sua sobrevivência que vão além dos cuidados requeridos por outros adolescentes. Objetivo: Apresentar a construção do discurso emancipatório de adolescentes com necessidades especiais de saúde a respeito das possibilidades de viver a adolescência com demandas de cuidados. Método: Pesquisa de abordagem qualitativa, descritiva e exploratória. A produção de dados foi mediada pela leitura de prontuários, entrevista semiestruturada e construção de genogramas e ecomapas de 35 adolescentes atendidos no ambulatório de especialidades. As enunciações foram gravadas em mídia digital, transcritas e submetidas à análise de discurso francesa de Pêcheux. Resultados: Os discursos representam um conjunto de atitudes capazes de romper com um padrão imposto pela família e pelos profissionais de saúde, em que os adolescentes são vistos somente pelas demandas de cuidados de saúde. Conclusões: Os adolescentes almejam ser vistos por suas possibilidades, como sujeitos no mundo e não apenas pelas demandas de cuidados para sua sobrevivência.
\end{abstract}

Palavras-chave: Discurso; Adolescente; Poder; Saúde da Pessoa com Deficiência; Pesquisa Qualitativa.

\section{Analysis of the Emancipatory Discourse on Adolescents with Special Health Needs}

Abstract: Introduction: Adolescents with special health needs (CRIANES) need health care for their survival that goes beyond the care required by other adolescents. Objective: To present the construction of the emancipatory discourse of adolescents with special health needs regarding the possibilities of experiencing adolescence with care demands. Method: Study of a qualitative approach developed through the reading of medical records, interview guided by a semi-structured script and construction of genograms and ecomaps of 35 adolescents. The speeches were recorded, transcribed and submitted to Pêcheux's speech analysis. Results: The speeches represent a set of attitudes capable of breaking with a pattern imposed by the family and health professionals, in which adolescents are seen by the demands of health care. Conclusions: Adolescents want to be seen for their possibilities, as subjects in the world and not just for the demands of care for their survival.

Keywords: Address; Adolescent; Power; Health of the Disabled; Qualitative Research.

\section{Introdução}

Os adolescentes com necessidades especiais de saúde (CRIANES) demandam de cuidados de saúde para sua sobrevivência que vão além dos cuidados requeridos por outros adolescentes, passam por repetidas hospitalizações e percorrem uma longa trajetória em busca de atenção/cuidados para suas necessidades especiais de saúde.

Considerando a trajetória de vida das CRIANES para a manutenção de seus cuidados de saúde e sobrevivência, acredita-se que o adolescente que vive com algum tipo de necessidade especial de saúde deve assumir o papel de sujeito de sua história, imerso em suas matrizes histórico-sócio-culturais. Assim, ao aplicar o referencial teórico de Paulo Freire é possível olhar para o cotidiano de cuidado, com as lentes da prática dialógica, como um dos inúmeros caminhos para negociar saberes e práticas (Freire, 2014). 
A partir do olhar freiriano sobre o opressor e o oprimido, pode-se pensar na realidade das CRIANES na adolescência, não com o intuito de estigmatizá-las, mas com a intenção de que sejam vistas como indivíduos que devem ter suas raízes, seus aspectos individuais e sociais respeitados. Torna-se possível compreender que o adolescente deve sair da condição de oprimido, e isso é possível ao enfrentar a realidade e se reconhecer como sujeito no mundo. Para Freire (2014), o oprimido deve reconhecer a situação de oprimido, e buscar a mudança e a transformação social. O oprimido vive numa situação de impotência diante do opressor, pois ao tentar ser mais, o oprimido não deve assumir a posição de opressor.

$\mathrm{Na}$ perspectiva da pedagogia libertadora de Freire, o homem oprimido desvela o mundo opressor e se compromete a transformá-lo, na busca pela igualdade social. O processo de libertação não está atrelado a ativismo, mas à reflexão para a transformação. Diferente da educação bancária, em que a educação está voltada à transmissão de conteúdo sem contestá-lo. Nesse sentido, a educação bancária é um instrumento de opressão, e para romper com este ciclo é necessário romper o sistema que gera a desigualdade social entre as pessoas (Freire, 2014; Freire, 2011).

A partir das lentes de Freire entende-se que, ninguém ignora tudo, ninguém sabe tudo, todos sabem alguma coisa, todos ignoram alguma coisa, por isso é possível aprender sempre (Freire, 2014). Essas reflexões também podem ser aplicadas na saúde do adolescente, sobretudo aos que vivem com algum tipo de necessidade especial de saúde. Muitas vezes, devido ao seu estado de saúde cronificado e a extensa rede institucional para suprir suas demandas de cuidados, as CRIANES podem viver na condição de oprimidos. Nesse sentido, é fundamental que os profissionais de saúde, entre eles os de enfermagem, estejam preparados para acolher esses adolescentes, quebrando a cultura do silêncio, oportunizando-Ihes uma participação ativa em seu processo de cuidado.

Ao pensar na saúde da CRIANES na adolescência é fundamental reconhecer que se fazem emergentes serviços de saúde para atender as demandas de cuidado dessa população, além da possibilidade de oportunizar que os adolescentes falem sobre sua condição de saúde e cotidiano de cuidados.

Frente ao exposto, questiona-se: Como é o cotidiano de cuidados dos adolescentes que vivem com necessidades especiais de saúde?

Este estudo objetiva apresentar a construção do discurso emancipatório de adolescentes com necessidades especiais de saúde a respeito das possibilidades de viver a adolescência com demandas de cuidados.

\section{Metodologia}

Trata-se de uma pesquisa com abordagem qualitativa, tipo exploratória e descritiva realizada no espaço temporal entre os anos de 2016 e 2017.

O cenário da pesquisa foi o ambulatório de especialidades que atende a população de CRIANES em um hospital de ensino no sul do Brasil, referência em atendimentos pelo Sistema Único de Saúde (SUS).

Para selecionar os participantes utilizou-se a leitura preliminar de prontuários de adolescentes atendidos no ambulatório, sempre no turno anterior à consulta, a fim de identificar se os adolescentes em atendimento correspondiam a população de CRIANES.

Participaram da pesquisa 35 CRIANES entre 12 e 18 anos de idade, que conhecessem sua necessidade especial de saúde, com condições cognitivas e para verbalizar. A produção de dados ocorreu em sala anexa ao ambulatório de especialidades, no turno da consulta de saúde dos adolescentes.

A produção de dados qualitativos foi desenvolvida por meio de entrevista semiestruturada, constituída por um roteiro composto por questões referentes ao cotidiano de cuidado de adolescentes. As entrevistas foram gravadas em mídia digital, com duração média entre 20 e 25 minutos. 
De acordo com Moreira \& Caleffe (2008), a produção de dados qualitativos através de entrevista semiestruturada possibilita exercer um certo controle na conversação, todavia mantém a liberdade de expressão dos entrevistados e a possibilidade de aprofundar temáticas que são pertinentes. Para a realização de uma entrevista semiestruturada, deve ser utilizado um "protocolo" ou "guia de entrevista" que remete ao conjunto de perguntas que orientam o pesquisador.

Ao final de cada entrevista utilizou-se a construção de genograma e ecomapa guiado pelo adolescente entrevistado, com o intuito de demonstrar o cotidiano de cuidado dessas CRIANES e os pontos fortes, moderados e fracos que compõe este processo. O genograma representa a composição familiar interna, evidenciando os núcleos de cuidado, dos quais participam diferentes pessoas. No genograma são utilizados símbolos que demonstram os membros da família, suas características e os diferentes tipos de relação. O ecomapa representa a visão geral com os recursos acessados ou até mesmo pelas privações. Nesta construção, é possível elucidar também as redes de relações, que podem ser representadas pelos amigos, vizinhos e redes sociais. As linhas mais fortes expressam a intesidade do vínculo existente e as linhas cortadas elucidam relações estressantes (Wright \& Leahey, 2008).

As enunciações passaram pela dupla transcrição no Programa Microsoft Word, realizadas pela pesquisadora e auxiliar de pesquisa, e foram submetidas à análise de discurso (AD) na corrente francesa de Pêcheux. A AD possibilita o movimento discursivo, desperta a memória latente dos participantes e as formas de produção de sentido (Orlandi, 2015). Para demonstrar o movimento no discurso dos adolescentes participantes, a pesquisadora criou uma legenda com recursos ortográficos do Word, para demonstrar aos leitores os movimentos das enunciações: /: pausa curta; //: pausa longa; ///: pausa muito longa; ...: pensamento incompleto; \#: interrupção; [...]: recorte da enunciação e (texto): comentários da pesquisadora.

Em seguida, realizou-se a leitura do texto com enfoque na posição discursiva do sujeito, legitimada pela união da dimensão social, histórica e ideológica. Para esta etapa utilizouse os dispositivos analíticos: o silenciamento, o ocultamento, a formação imaginária, a polissemia, o interdiscurso, a paráfrase e a metáfora. Outro recurso utilizado foi a análise cromática, a fim de identificar os efeitos de sentido dos discursos transcritos. Por fim, as enunciações foram alocadas em um quadro onde foram inseridos comentários analíticos que subsidiaram temas geradores e comentários analíticos.

A pesquisa seguiu a resolução $466 / 2012$ que trata dos aspectos éticos sobre a pesquisa com seres humanos no Brasil, e teve início após receber a aprovação pelo Comitê de Ética em Pesquisa da Universidade Federal de Santa Maria, com número de CAAE 57774916.7.0000.5346, e parecer número 1.673.887. Aos responsáveis pelos adolescentes foi entregue o termo de consentimento livre e esclarecido e, para os adolescentes o termo de assentimento, ambos em duas vias.

\section{Resultados}

A análise de discurso das 35 entrevistas revelou o discurso emancipatório de adolescentes que vivem com necessidades especiais de saúde. Os adolesecntes utilizaram expressões: "A adolescência é normal", "Consigo fazer tudo" e "Eu me cuido" simbolizando o processo de emancipação para o cuidado, em que as CRIANES não se encontram na posição de submissão devido a sua condição de saúde, mas que buscam o reconhecimento de serem adolescentes no mundo, por meio das suas possibilidades.

As CRIANES participantes atribuíram significados diversos sobre o vivido da adolescência com demandas de cuidados de saúde por meio das enunciações: "É assim desde pequeno, eu fui aprendendo a me cuidar", "É normal, quando eu vejo que não posso comer alguma coisa eu levo meu lanche separado", "Já me acostumei com o chapelão e com os cuidados com a minha pele, para meus amigos é normal", "Eu tenho amigos da igreja, adoro ir à igreja, sair com eles". As enunciações revelam que para os adolescentes é importante aceitar as demandas de cuidado com naturalidade. 
Observa-se também, que muitos adolescentes desenvolvem cuidados de saúde desde a infância, sendo este incorporado ao processo natural de vida.

Por meio das falas das CRIANES, observa-se que elas assumem o protagonismo de seu cuidado, como pode ser visto nos discursos a seguir: "Eu sempre tive o apoio da mãe e do pai, mas hoje eu me cuido sozinho", "Eu prefiro que ninguém saiba, não sou diferente", "Tudo normal, eu me conheço, se não me sentir bem eu sei o que fazer", "Desde pequena eu já sabia o que eu tinha, pra mim é natural". Os discursos revelam que o cuidado no cotidiano começa na infância, e que a família participa do cuidado. Ao chegar na adolescência, os adolescentes sinalizam para a emancipação e rejeitam a opressão que a necessidade especial de saúde lhes impõem.

Para cada entrevista foi utilizada a construção de genogramas e ecomapas, que elucidaram a conformação das redes de apoio e cuidado desses adolescentes. Por meio dos genogramas e ecomapas, as CRIANES apresentaram uma ampla rede institucional, composta pelos serviços de saúde, ambulatório de especialidades, hospital, atenção primária em alguns casos, além do apoio recebido pela escola e igreja. Para os adolescentes, os cuidados de saúde estão mais centrados no gênero feminino através da mãe, avós, tias e madrinhas.

Os adolescentes participantes deste estudo apresentam inquietações a respeito do viver a adolescência com autonomia, sobre o poder de escolhas, sobre os cuidados muitas vezes internalizado em discursos de familiares e nas orientações restritivas: "Sempre me avisam que tem que se cuidar", "Ah, não pode fazer isso, não pode fazer aquilo", "A mãe sempre fala, tá sempre em cima", "Nem sempre é como eu quero, tem coisas que não dá pra fazer". Essas enunciações indicam o desejo pela autonomia, e que apesar do zelo dos familiares, os adolescentes almejam serem reconhecidos com naturalidade e não apenas pelas demandas de cuidados.

Os achados deste estudo indicaram a premência da inclusão e fortalecimento desta temática no processo formativo em Enfermagem e Saúde, assim como suscita outras propostas de pesquisa e extensão que trabalhem com a CRIANES na fase da adolescência. A fonte de apoio para a emancipação dos adolescentes deste estudo revelou significados maiores ao cotidiano de cuidado desses adolescentes, onde a complexidade de cuidados requeridos não é maior que o desejo de vivenciarem a adolescência com naturalidade assumindo o protagonismo do seu cuidado.

\section{Discussão}

As CRIANES participantes deste estudo, desenvolvem uma reflexão crítica sobre si mesmo, na busca pelo ser mais. Para Freire (2008), a reflexão crítica sobre a existência, possibilita ao homem ser mais. Nesta perspectiva, ninguém educa ninguém, o homem deve ser sujeito de sua própria educação, não pode ser o objeto dela (Freire, 2014). De acordo com Faial, Costa, Silva, Pereira \& Faial (2019) os adolescentes são questionadores, indagam e apresentam inquietação ao viver o adolescer. Esses aspectos fazem parte do processo de autoafirmação e de descobrimento sobre si mesmo, e suas possibilidades.

Destaca-se ainda, que para a população adolescente os grupos, as amizades, a escola e a socialização são fundamentais nesta fase da vida. Para Faial, Costa, Silva, Pereira \& Faial (2019), os adolescentes apresentam a necessidade de verbalizar sobre seus sentimentos, seu vivido, a satisfação e a insatisfação desta fase da vida. Na adolescência é comum a busca por respostas, inclusive pelo desconforto e o conflito que os adolescentes vivenciam durante o seu processo de desenvolvimento. 
Os achados deste estudo remetem sobre o reconhecimento de ser adolescente, sem estigmas, independente das demandas de cuidados apresentadas. Segundo Fernandes, Speranza, Mazak, Gasparini \& Cid (2021), os adolescentes que vivem com necessidades especiais têm uma ampla rede institucional, são necessárias diferentes estratégias de cuidado que busquem apoiar as particularidades de cada adolescente, possibilitando espaços de cuidado por meio do respeito, empatia e solidariedade. Contextualizando os achados com este estudo, observa-se que é fundamental possibilitar ao adolescente conhecer, refletir, questionar sobre seus cuidados e condições de saúde.

$O$ processo de cuidado de adolescentes perpassa pelas possibilidades e habilidades em diferentes níveis emancipatórios, em que a enfermagem contribui para a troca de saberes sobre esses cuidados, para as práticas de educação em saúde e possibilidades para a reflexão. Assim, é possível que as CRIANES transitem de uma consciência ingênua para crítica sobre seus cuidados de saúde no cotidiano (Silveira \& Neves, 2019).

Os resultados apontam o protagonismo dos adolescentes em seu processo de cuidado $e$ a busca pela emancipação, mesmo diante das orientações e cuidados familiares. A emancipação revela um processo de libertação nos diferentes campos que compõem o cotidiano do homem oprimido: político, cultural, humano e social. A libertação nasce para que o homem deixe de ser oprimido (Freire, 2014). O caminho da emancipação por meio do resgate de sua verdadeira humanização é tarefa histórica do ser humano e está situada num contexto social e objetivo concreto, envolvendo pessoas e comunidades (Ambrosini, 2012).

Em relação a naturalidade que os adolescentes apresentam suas demandas de cuidados, esse processo vem amparado pelos cuidados recebidos desde a infância. Tratar com naturalidade as necessidades especiais de saúde e manter um atendimento singular, que priorize a melhoria de sua qualidade de vida é um direito. Em que a saúde desses adolescentes deve ser uma prioridade nas políticas públicas de saúde, com profissionais qualificados, amparado pelos pressupostos da humanização e com foco no cuidado integral (Silva, Góes, Vasconcelos, Jamelli, Eickmann, Melo \& Lima, 2020).

Os genogramas e ecomapas revelam uma ampla rede institucional e o cuidado centrado principalmente, nas mulheres da família, em que às mães desempenham essa função (Neves, Silveira, Arrué, Pieszak, Zamberlan \& Santos, 2015). As mães de CRIANES, muitas mulheres abdicam de sua vida pessoal em detrimento do cuidado de seus filhos. Há reflexões sobre diferenças de gênero, resiliência, amor, bem-estar integral, convivência, respeito, família, escola, sociedade e rede de apoio, do ponto de vista do cuidador. As mães de CRIANES possuem uma potência afetiva, social e cultural na luta pela sobrevivência de seus filhos e pelos espaços de inclusão social (Cavalcante, Lau, Barbosa, Berlim, Menezes, Braga, Amorim \& Amorim, 2016).

Este estudo revelou uma ampla rede institucional em prol do cuidado especializado dos adolescentes. Esses achados são convergentes com estudo publicado por Neves, Silveira, Arrué, Pieszak, Zamberlan \& Santos (2015), em que as CRIANES e seus familiares deparam-se com dificuldades de acesso aos serviços de saúde, as necessidades de saúde complexas, hospitalizações frequentes e a necessidade de diferentes profissionais e especialidades de saúde para suprir as demandas de cuidados. Considerando as necessidades de cuidado das CRIANES, mesmo em situações adversas, é essencial que a enfermagem esteja preparada e capacitada para atendê-las (Inácio \& Peixoto, 2018).

Assim, acredita-se que as CRIANES na fase da adolescência devem ser reconhecidas por suas possibilidades, como sujeitos no mundo e não apenas pelas demandas de cuidados requeridos para sua sobrevivência. 


\section{Conclusões}

O discurso dos adolescentes participantes deste estudo representa um conjunto de atitudes capazes de romper com estigmas, restrições familiares e tabus sociais. As CRIANES deste estudo almejam viver a adolescência com naturalidade e autonomia. Por meio do discurso emancipatório rejeitam a opressão que a necessidade especial de saúde Ihes impõe, e desejam ser vistas para além de suas demandas de cuidados.

Para esta pesquisa, a utilização da entrevista semiestruturada e a construção de genogramas e ecomapas, bem como a utilização da AD demonstraram-se favoráveis para olhar com sensibilidade e profundidade para os movimentos emancipatórios dos adolescentes.

Quanto as limitações deste estudo, destaca-se que o mesmo foi realizado em um ambulatório de especialidades, não sendo possível generalizações ou comparações com outros serviços de saúde que atendem a saúde do adolescente. Por fim, sugere-se a realização de pesquisas qualitativas que possibilitem aos adolescentes espaços de diálogo e escuta.

\section{Referências}

Cavalcante,F.G., Lau, L.F., Barbosa, G.F., Berlim, D.L.G., Menezes, N.C., Braga, D.C., Amorim A.C. \& Amorim, Y.C. (2016). Impactos de um documentário sobre o cotidiano de mães e filhos com deficiência: uma análise de cinedebates. Ciência \& Saúde, 21(10).

Faial, L.C.M., Costa, R.M., Silva, R.A., Pereira, E.R. \& Faial, C.S.G. (2019). A saúde na escola: percepções do ser adolescente. Rev. Bras. Enferm., 72 (4).

Fernandes, A.D.S.A., Speranza, M., Mazak, M.S.R., Gasparini, D.A. \& Cid, M.F.B. (2021). Desafios cotidianos e possibilidades de cuidado com crianças e adolescentes com Transtorno do Espectro Autista (TEA) frente à COVID-19. Cadernos Brasileiros de Terapia Ocupacional, 29:e2121.

Freire, P. (2008). Conscientização, teoria e prática da libertação: uma introdução ao pensamento de Paulo Freire. $3^{\text {a }}$. ed. São Paulo: Centauro Editora.

Freire, P. (2011). Pedagogia da autonomia: saberes necessários à prática educativa. 43á. ed. São Paulo: Paz e Terra.

Freire, P. (2014). Pedagogia do oprimido. 56로. ed. Rio de Janeiro: Paz e Terra.

Inácio, A..LR., Peixoto, A.P.G.L. (2017). A assistência de enfermagem e o cuidado familiar às crianças com necessidades especiais de saúde: uma revisão integrativa. Rev Atenção Saúde, 15(53):87-94.

Moreira, H. \& Caleffe, L. G. (2008). Metodologia científica para o professor pesquisador. $2^{\text {a }}$. ed. Rio de Janeiro: Lamparina.

Wright, L. M. \& Leahey, M. (2008). Enfermeiras e famílias: um guia para avaliação e intervenção na família. 4⿳亠丷. ed. São Paulo: Roca.

Machado, A.C.C., Rossi, C.S., Rodrigues, B.M.R.D., Pacheco, S.T.A., Arantes, P.C.C. \& Rodrigues, B.R.D. (2016). Análise de discurso nas pesquisas de enfermagem na saúde da criança e do adolescente. Rev enferm UERJ, 24(6), e25737.

Neves, E.T., Silveira, A., Arrué, A.M., Pieszak, G.M., Zamberlan, K.C., \& Santos, R.P. (2015). Rede de cuidados de crianças com necessidades especiais de saúde. Texto contexto - enferm. 24(2).

Orlandi, E.P. (2015). Análise do discurso: princípios e procedimentos. $12^{\mathrm{a}}$ ed. Campinas (SP): Pontes Editores.

Silva, E.L.M.S., Góes, P.S.A., Vasconcelos, M.M.V.B., Jamelli, S.R., Eickmann, S.H., Melo, M.M.D.C. \& Lima, M.C. (2020). Cuidados em saúde bucal a crianças e adolescentes com paralisia cerebral: percepção de pais e cuidadores. Ciência \& Saúde Coletiva 25(10).

Silveira, A. \& Neves, E.T. (2019). Cotidiano de cuidado de adolescentes com necessidades especiais de atenção à saúde. Acta Paulista de Enfermagem 32(3). 